\title{
PENYELESAIAN SISTEM DESKRIPTOR LINIER DISKRIT BEBAS WAKTU DENGAN MENGGUNAKAN METODE DEKOMPOSISI KANONIK
}

\author{
USWATUN HASANAH \\ Program Studi Matematika, \\ Fakultas Matematika dan Ilmu Pengetahuan Alam, Universitas Andalas Padang, \\ Kampus UNAND Limau Manis Padang, Indonesia, \\ hasan.uswatunhasanah@gmail.com
}

\begin{abstract}
The linear discrete descriptor systems are used in many applications, especially in mathematical modeling for biology, economics and electrical engineering. In this paper, the solving of invariant time linear discrete descriptor systems using the canonical decomposition method was studied. The canonical decomposition method reduces the systems under consideration into two simple subsystems. The solution is obtained from two simple subsystems. An example is given to illustrate this method.
\end{abstract}

Kata Kunci: Linear discrete descriptor systems, canonical decomposition method.

\section{Pendahuluan}

Diberikan sistem deskriptor linier diskrit berikut:

$$
E \mathbf{x}(k+1)=A \mathbf{x}(k)+B \mathbf{u}(k), \quad \mathbf{x}(0)=\mathbf{x}_{0},
$$

di mana $E, A \in \mathbb{R}^{n \times n}, B \in \mathbb{R}^{n \times m}$, dan $k \in \mathbb{Z}_{+}$. Dalam sistem deskriptor linier (1.1), $\mathbf{x}(k) \in \mathbb{R}^{n}$ menyatakan vektor keadaan (state), $\mathbf{u}(k) \in \mathbb{R}^{m}$ menyatakan vektor input (kontrol), dan $k$ menyatakan waktu.

Hal yang menarik untuk dikaji dari sistem deskriptor linier diskrit bebas waktu adalah penentuan solusi dari sistem (1.1) apabila $E$ adalah matriks singular.

Sistem (1.1) dikatakan regular apabila $\operatorname{det}(\gamma E-A) \neq 0$ untuk suatu $\gamma \in \mathbb{R}$. Sistem regular (1.1) dapat diselesaikan dengan banyak metode. Dalam [2], sistem regular (1.1) diselesaikan dengan menggunakan invers Drazin dan dengan mengambil transformasi $z$ terhadap sistem (1.1) tersebut. Dalam tulisan ini akan dikaji tentang penyelesaian sistem deskriptor linier diskrit regular (1.1) dengan $E$ adalah singular dengan menggunakan metode dekomposisi kanonik, yang diturunkan dari penyelesaian sistem deskriptor linier kontinu regular sebagaimana yang telah dikaji oleh Yip and Sincovec [3]. 


\section{Penyelesaian Sistem Deskriptor Linier Diskrit Bebas Waktu dengan Menggunakan Metode Dekomposisi Kanonik}

Teorema 2.1. [1] Sistem (1.1) dikatakan regular jika dan hanya jika terdapat matriks nonsingular $Q, P \in \mathbb{R}^{n \times n}$ sedemikian sehingga

$$
Q E P=\left(\begin{array}{cc}
I_{n_{1}} & 0 \\
0 & N
\end{array}\right) \text { dan } Q A P=\left(\begin{array}{cc}
A_{1} & 0 \\
0 & I_{n_{2}}
\end{array}\right),
$$

di mana $A_{1} \in \mathbb{R}^{n_{1} \times n_{1}}, N \in \mathbb{R}^{n_{2} \times n_{2}}, n_{1}+n_{2}=n$, dan matriks $N$ adalah nilpotent dengan indeks nilpotensi $h$.

Bukti. $(\Rightarrow)$ Misalkan sistem (1.1) regular. Maka $\operatorname{det}(\gamma E-A) \neq 0$ untuk suatu $\gamma \in \mathbb{R}$, sehingga $(\gamma E-A)^{-1}$ ada. Misalkan

$$
\hat{E}=(\gamma E-A)^{-1} E \text { dan } \hat{A}=(\gamma E-A)^{-1} A .
$$

Maka

$$
\begin{aligned}
\hat{A} & =(\gamma E-A)^{-1}(\gamma E+A-\gamma E) \\
& =(\gamma E-A)^{-1}(-(\gamma E-A)+\gamma E) \\
& =-I+\gamma(\gamma E-A)^{-1} E \\
& =\gamma \hat{E}-I .
\end{aligned}
$$

Karena $E$ singular, maka $\hat{E}$ juga singular. Akibatnya, terdapat matriks nonsingular $T \in \mathbb{R}$ sedemikian sehingga

$$
T^{-1} \hat{E} T=\left(\begin{array}{cc}
E_{1} & 0 \\
0 & E_{2}
\end{array}\right)
$$

di mana $E_{1} \in \mathbb{R}^{n_{1} \times n_{1}}$ adalah nonsingular dan $E_{2} \in \mathbb{R}^{n_{2} \times n_{2}}$ adalah nilpotent. Akibatnya, $\left(\gamma E_{2}-I\right)$ adalah nonsingular. Perhatikan kesamaan berikut

$$
(\alpha E-A)=(\alpha-\gamma) E+(\gamma E-A)
$$

untuk suatu $\alpha \in \mathbb{R}$. Kalikan kedua ruas kesamaan $(2.2)$ dengan $(\gamma E-A)^{-1}$. Maka diperoleh

$$
\begin{aligned}
(\gamma E-A)^{-1}(\alpha E-A) & =(\alpha-\gamma)(\gamma E-A)^{-1} E+I \\
& =(\alpha-\gamma) \hat{E}+I \\
& =(\alpha-\gamma) T\left(\begin{array}{cc}
E_{1} & 0 \\
0 & E_{2}
\end{array}\right) T^{-1}+I .
\end{aligned}
$$

Dapat dilihat bahwa (2.3) ekivalen dengan

$$
\begin{aligned}
T^{-1}(\gamma E-A)^{-1}(\alpha E-A) T & =(\alpha-\gamma)\left(\begin{array}{cc}
E_{1} & 0 \\
0 & E_{2}
\end{array}\right)+I \\
& =\left(\begin{array}{cc}
(\alpha-\gamma) E_{1}+I_{n_{1}} & 0 \\
0 & (\alpha-\gamma) E_{2}+I_{n_{2}}
\end{array}\right) \\
& =\left(\begin{array}{cc}
(\alpha-\gamma) E_{1}+I_{n_{1}} & 0 \\
0 & -\left(\gamma E_{2}-I_{n_{2}}\right)+\alpha E_{2}
\end{array}\right) .
\end{aligned}
$$


Selanjutnya kalikan kedua ruas (2.4) dengan

$$
\left(\begin{array}{cc}
E_{1}^{-1} & 0 \\
0 & \left(\gamma E_{2}-I_{n_{2}}\right)^{-1}
\end{array}\right)
$$

sehingga diperoleh

$$
\begin{aligned}
& \left(\begin{array}{cc}
E_{1}^{-1} & 0 \\
0 & \left(\gamma E_{2}-I_{n_{2}}\right)^{-1}
\end{array}\right) T^{-1}(\gamma E-A)^{-1}(\alpha E-A) T \\
& \quad=\left(\begin{array}{cc}
E_{1}^{-1} & 0 \\
0 & \left(\gamma E_{2}-I_{n_{2}}\right)^{-1}
\end{array}\right)\left(\begin{array}{cc}
(\alpha-\gamma) E_{1}+I_{n_{1}} & 0 \\
0 & -\left(\gamma E_{2}-I_{n_{2}}\right)+\alpha E_{2}
\end{array}\right) \\
& \quad=\left(\begin{array}{cc}
(\alpha-\gamma) I_{n_{1}}+E_{1}^{-1} & 0 \\
0 & \alpha\left(\gamma E_{2}-I_{n_{2}}\right)^{-1} E_{2}-I_{n_{2}}
\end{array}\right) \\
& \quad=\alpha\left(\begin{array}{ccc}
I_{n_{1}} & 0 & \\
0 & \left(\gamma E_{2}-I_{n_{2}}\right)^{-1} E_{2}
\end{array}\right)-\left(\begin{array}{cc}
\gamma I_{n_{1}}-E_{1}^{-1} & 0 \\
0 & I_{n_{2}}
\end{array}\right) .
\end{aligned}
$$

Tuliskan

$$
\left(\begin{array}{cc}
E_{1}^{-1} & 0 \\
0 & \left(\gamma E_{2}-I_{n_{2}}\right)^{-1}
\end{array}\right) T^{-1}(\gamma E-A)^{-1}=Q \text { dan } T=P .
$$

Dari (2.5) diperoleh

$$
Q(\alpha E-A) P=\alpha\left(\begin{array}{cc}
I_{n_{1}} & 0 \\
0 & N
\end{array}\right)-\left(\begin{array}{cc}
A_{1} & 0 \\
0 & I_{n_{2}}
\end{array}\right)
$$

di mana $N=\left(\gamma E_{2}-I_{n_{2}}\right)^{-1} E_{2}$ dan $A_{1}=\gamma I_{n_{1}}-E_{1}^{-1}$. Jadi, diperoleh $Q$ dan $P$, sehingga (2.1) berlaku.

$(\Leftarrow)$ Misalkan terdapat matriks nonsingular $Q, P \in \mathbb{R}$ sedemikian sehingga $(2.1)$ berlaku untuk suatu $A_{1} \in \mathbb{R}^{n_{1} \times n_{1}}, N \in \mathbb{R}^{n_{2} \times n_{2}}, n_{1}+n_{2}=n$, dan matriks $N$ adalah nilpotent dengan indeks nilpotensi $h$. Misalkan $\gamma \notin \sigma\left(A_{1}\right)$. Tanpa mengurangi keumuman, misalkan $\gamma \in \mathbb{R}$. Maka $\operatorname{det}\left(\gamma I_{n_{1}}-A_{1}\right) \neq 0$. Tuliskan

$$
\operatorname{det}\left(\gamma N-I_{n_{2}}\right)=(-1)^{n_{2}} .
$$

Selanjutnya

$$
\begin{aligned}
\operatorname{det}(\gamma E-A) & =\operatorname{det}\left(\gamma Q^{-1} Q E P P^{-1}-Q^{-1} Q A P P^{-1}\right) \\
& =\operatorname{det}\left(Q^{-1}\right) \operatorname{det}(\gamma Q E P-Q A P) \operatorname{det}\left(P^{-1}\right) \\
& =\operatorname{det}\left(Q^{-1}\right) \operatorname{det}\left(\gamma\left(\begin{array}{cc}
I_{n_{1}} & 0 \\
0 & N
\end{array}\right)-\left(\begin{array}{cc}
A_{1} & 0 \\
0 & I_{n_{2}}
\end{array}\right)\right) \operatorname{det}\left(P^{-1}\right)
\end{aligned}
$$

Karena (2.6), maka (2.7) dapat ditulis sebagai berikut

$$
\begin{aligned}
\operatorname{det}(\gamma E-A) & =\operatorname{det}\left(Q^{-1}\right) \operatorname{det}\left(\gamma I_{n_{1}}-A_{1}\right)(-1)^{n_{2}} \operatorname{det}\left(P^{-1}\right) \\
& =(-1)^{n_{2}} \operatorname{det}\left(Q^{-1}\right) \operatorname{det}\left(\gamma I_{n_{1}}-A_{1}\right) \operatorname{det}\left(P^{-1}\right) \\
& \neq 0 .
\end{aligned}
$$

Akibatnya, sistem (1.1) adalah regular. 
Perhatikan kembali sistem deskriptor regular (1.1). Selanjutnya misalkan $\mathbf{x}(k)=$ $P \mathbf{y}(k)$ dan $Q B=\left(\begin{array}{c}B_{1} \\ B_{2}\end{array}\right)$ untuk suatu $Q, P$ adalah nonsingular, dan $\mathbf{y} \in \mathbb{R}^{n}$. Maka sistem (1.1) dapat ditulis menjadi

$$
Q E P \mathbf{y}(k+1)=Q A P \mathbf{y}(k)+\left(\begin{array}{c}
B_{1} \\
B_{2}
\end{array}\right) \mathbf{u}(k) .
$$

Dengan menggunakan Teorema 1, maka sistem (1.1) dapat ditulis menjadi

$$
\left(\begin{array}{cc}
I_{n_{1}} & 0 \\
0 & N
\end{array}\right) \mathbf{y}(k+1)=\left(\begin{array}{cc}
A_{1} & 0 \\
0 & I_{n_{2}}
\end{array}\right) \mathbf{y}(k)+\left(\begin{array}{c}
B_{1} \\
B_{2}
\end{array}\right) \mathbf{u}(k)
$$

dengan syarat awal $\mathbf{y}(0)=P^{-1} \mathbf{x}(0)$. Hubungan (2.8) memperlihatkan bahwa sistem (1.1) dapat direduksi menjadi dua subsistem, yaitu

$$
\mathbf{y}_{1}(k+1)=A_{1} \mathbf{y}_{1}(k)+B_{1} \mathbf{u}(k), \mathbf{y}_{1}(0)=\mathbf{y}_{10}
$$

dan

$$
N \mathbf{y}_{2}(k+1)=\mathbf{y}_{2}(k)+B_{2} \mathbf{u}(k), \mathbf{y}_{2}(0)=\mathbf{y}_{20},
$$

di mana $\mathbf{y}_{1} \in \mathbb{R}^{n_{1}}$ dan $\mathbf{y}_{2} \in \mathbb{R}^{n_{2}}$.

Teorema 2.2. [1] Untuk sebarang vektor input $\mathbf{u}(k)$, solusi subsistem (2.9) adalah tunggal dan solusinya diberikan oleh

$$
\mathbf{y}_{1}(k)=A_{1}^{k} \mathbf{y}_{1}(0)+\sum_{j=0}^{k-1} A_{1}^{k-j-1} B_{1} \mathbf{u}(j) .
$$

Selanjutnya dengan mengambil transformasi $z$ dari persamaan (2.9) diperoleh

$$
\mathbf{Y}_{1}(z)=\left(z I-A_{1}\right)^{-1} z \mathbf{y}_{1}(0)+\left(z I-A_{1}\right)^{-1} B_{1} \mathbf{U}(z) .
$$

Dengan mengambil transformasi $z$ invers dari persamaan (2.12) diperoleh

$$
\mathbf{y}_{1}(k)=\mathcal{Z}^{-1}\left[\left(z I-A_{1}\right)^{-1} z\right] \mathbf{y}_{1}(0)+\mathcal{Z}^{-1}\left[\left(z I-A_{1}\right)^{-1} B_{1} \mathbf{U}(z)\right] .
$$

Dengan membandingkan persamaan (2.11) dengan (2.13) diperoleh

$$
A_{1}^{k}=\mathcal{Z}^{-1}\left[\left(z I-A_{1}\right)^{-1} z\right]
$$

dan

$$
\sum_{j=0}^{k-1} A_{1}^{k-j-1} B_{1} \mathbf{u}(j)=\mathcal{Z}^{-1}\left[\left(z I-A_{1}\right)^{-1} B_{1} \mathbf{U}(z)\right] .
$$

Teorema 2.3. [1] Untuk sebarang vektor input $\mathbf{u}(k)$, solusi subsistem (2.10) adalah tunggal, yaitu

$$
\mathbf{y}_{2}(k)=-\sum_{j=0}^{h-1} N^{j} B_{2} \mathbf{u}(k+j), \quad k \in \mathbb{Z}_{+} .
$$


Dari (2.11) dan (2.14), diperoleh solusi dari sistem (1.1), yaitu

$$
\mathbf{x}(k)=P\left(\begin{array}{c}
A_{1}^{k} \mathbf{y}_{1}(0)+\sum_{j=0}^{k-1} A_{1}^{k-j-1} B_{1} \mathbf{u}(j) \\
-\sum_{j=0}^{h-1} N^{j} B_{2} \mathbf{u}(k+j)
\end{array}\right) .
$$

Berdasarkan Teorema 1, 2, 3, maka dapat diuraikan prosedur untuk menentukan penyelesaian sistem (1.1), yaitu sebagai berikut:

(1) Periksa kekonsistenan kondisi awal $\mathbf{x}(0)=\mathbf{x}_{0}$ terhadap sistem (1.1).

(2) Periksa keregularan sistem (1.1) dengan memisalkan $\gamma \in \mathbb{R}$ sebagai suatu konstanta riil sebarang yang memenuhi $\operatorname{det}(\gamma E-A) \neq 0$.

(3) Tentukan $\hat{E}=(\gamma E-A)^{-1} E$.

(4) Tentukan matriks $T^{-1} \hat{E} T$ untuk memperoleh $E_{1}, E_{2}$, dan $T$ sedemikian sehingga $\hat{E}$ dan $T^{-1} \hat{E} T$ similar.

(5) Tentukan $Q$ dan $P$ dengan menggunakan (2.5) dan selanjutnya tentukan $Q E P$, $Q A P$, dan $Q B$ untuk memperoleh $A_{1}, N, B_{1}$, dan $B_{2}$.

(6) Misalkan didefinisikan sistem baru $\mathbf{x}(k)=P \mathbf{y}(k)$ dengan kondisi awal $\mathbf{y}(0)=$ $P^{-1} \mathbf{x}(0)$. Reduksi sistem baru tersebut menjadi dua subsistem, yaitu (2.9) dan (2.10), kemudian tentukan kondisi awal $\mathbf{y}_{1}(0)$ dan $\mathbf{y}_{2}(0)$ berturut-turut dari subsistem (2.9) dan (2.10).

(7) Tentukan penyelesaian dari subsistem (2.9) dan (2.10) tersebut berturut-turut dengan menggunakan (2.11) dan (2.14).

(8) Dari poin 7 yang telah diperoleh, tentukan sistem $\mathbf{x}(k)=P \mathbf{y}(k)$. Sistem $\mathbf{x}(k)$ yang diperoleh merupakan solusi akhir dari sistem (1.1).

\section{Contoh}

Contoh berikut mengilustrasikan bagaimana proses penyelesaian sistem deskriptor linier diskrit bebas waktu, di mana contoh yang diberikan merupakan sistem yang regular, kondisi awal $\mathbf{x}(0)=\mathbf{x}_{0}$ yang konsisten, dan vektor input berupa polinomial.

Diberikan sistem deskriptor linier diskrit

$$
E \mathbf{x}(k+1)=A \mathbf{x}(k)+B \mathbf{u}(k),
$$

dengan

$$
\begin{gathered}
E=\left(\begin{array}{llll}
1 & 0 & 0 & 0 \\
0 & 1 & 0 & 0 \\
0 & 0 & 1 & 0 \\
0 & 0 & 0 & 0
\end{array}\right), A=\left(\begin{array}{llll}
0 & 0 & 1 & 0 \\
1 & 0 & 0 & 0 \\
0 & 1 & 0 & 1 \\
0 & 0 & 1 & 0
\end{array}\right), B=\left(\begin{array}{ccc}
1 & 0 & 0 \\
1 & -1 & 2 \\
0 & 1 & 0 \\
0 & 0 & 1
\end{array}\right), \\
\mathbf{x}(0)=\left(\begin{array}{c}
-1 \\
-1 \\
-1 \\
-4
\end{array}\right), \operatorname{dan} \mathbf{u}(k)=\left(\begin{array}{c}
k^{2} \\
k+3 \\
k^{2}+1
\end{array}\right)
\end{gathered}
$$

untuk $k=0,1,2,3, \ldots$. Akan ditentukan solusi dari sistem (3.1) ini. Misalkan $\gamma=1$. Maka

$$
\operatorname{det}(\gamma E-A)-1 \neq 0
$$


Selanjutnya misalkan

$$
\hat{E}=(\gamma E-A)^{-1} E=\left(\begin{array}{cccc}
1 & 0 & 0 & 0 \\
1 & 1 & 0 & 0 \\
0 & 0 & 0 & 0 \\
-1 & -1 & -1 & 0
\end{array}\right)
$$

Karena setiap matriks bujursangkar similar dengan suatu matriks Jordan, maka terdapat matriks nonsingular $T \in \mathbb{R}^{n \times n}$ sedemikian sehingga

$$
T^{-1} \hat{E} T=\left(\begin{array}{cc}
E_{1} & 0 \\
0 & E_{2}
\end{array}\right)=\left(\begin{array}{llll}
1 & 1 & 0 & 0 \\
0 & 1 & 0 & 0 \\
0 & 0 & 0 & 1 \\
0 & 0 & 0 & 0
\end{array}\right)
$$

Dalam hal ini

$$
E_{1}=\left(\begin{array}{ll}
1 & 1 \\
0 & 1
\end{array}\right), E_{2}=\left(\begin{array}{ll}
0 & 1 \\
0 & 0
\end{array}\right), \operatorname{dan} T=\left(\begin{array}{cccc}
0 & 1 & 0 & 0 \\
1 & 0 & 0 & 0 \\
0 & 0 & 0 & 1 \\
-1 & 0 & -1 & 0
\end{array}\right) .
$$

Dengan menggunakan (2.5) diperoleh

$$
Q=\left(\begin{array}{cccc}
0 & 1 & 0 & 0 \\
1 & 0 & 0 & -1 \\
0 & 0 & -1 & 0 \\
0 & 0 & 0 & 1
\end{array}\right) \quad \text { dan } P=\left(\begin{array}{cccc}
0 & 1 & 0 & 0 \\
1 & 0 & 0 & 0 \\
0 & 0 & 0 & 1 \\
-1 & 0 & -1 & 0
\end{array}\right)
$$

Selanjutnya

$$
Q E P=\left(\begin{array}{cccc}
1 & 0 & 0 & 0 \\
0 & 1 & 0 & 0 \\
0 & 0 & 0 & -1 \\
0 & 0 & 0 & 0
\end{array}\right), Q A P=\left(\begin{array}{llll}
0 & 1 & 0 & 0 \\
0 & 0 & 0 & 0 \\
0 & 0 & 1 & 0 \\
0 & 0 & 0 & 1
\end{array}\right), \text { dan } Q B=\left(\begin{array}{ccc}
1 & -1 & 2 \\
1 & 0 & -1 \\
0 & -1 & 0 \\
0 & 0 & 1
\end{array}\right)
$$

sehingga diperoleh

$$
A_{1}=\left(\begin{array}{ll}
0 & 1 \\
0 & 0
\end{array}\right), N=\left(\begin{array}{cc}
0 & -1 \\
0 & 0
\end{array}\right), B_{1}=\left(\begin{array}{ccc}
1 & -1 & 2 \\
1 & 0 & -1
\end{array}\right), \text { dan } B_{2}=\left(\begin{array}{ccc}
0 & -1 & 0 \\
0 & 0 & 1
\end{array}\right) .
$$

Misalkan

$$
\mathbf{x}(k)=P \mathbf{y}(k)
$$

dengan

$$
\mathbf{y}(k)=\left(\begin{array}{l}
\mathbf{y}_{1}(k) \\
\mathbf{y}_{2}(k)
\end{array}\right) .
$$

Maka sistem (3.1) dapat ditulis menjadi

$$
\mathbf{y}_{1}(k+1)=\left(\begin{array}{ll}
0 & 1 \\
0 & 0
\end{array}\right) \mathbf{y}_{1}(k)+\left(\begin{array}{ccc}
1 & -1 & 2 \\
1 & 0 & -1
\end{array}\right)\left(\begin{array}{c}
k^{2} \\
k+3 \\
k^{2}+1
\end{array}\right)
$$


dan

$$
\left(\begin{array}{cc}
0 & -1 \\
0 & 0
\end{array}\right) \mathbf{y}_{2}(k+1)=\mathbf{y}_{2}(k)+\left(\begin{array}{ccc}
0 & -1 & 0 \\
0 & 0 & 1
\end{array}\right)\left(\begin{array}{c}
k^{2} \\
k+3 \\
k^{2}+1
\end{array}\right)
$$

dengan kondisi awal

$$
\mathbf{y}_{1}(0)=\left(\begin{array}{c}
-1 \\
-1
\end{array}\right) \text { dan } \mathbf{y}_{2}(0)=\left(\begin{array}{c}
5 \\
-1
\end{array}\right) \text {. }
$$

Dengan menggunakan (2.11), maka solusi sistem (3.2) adalah

$$
\begin{aligned}
\mathbf{y}_{1}(k) & =\left(\begin{array}{ll}
0 & 1 \\
0 & 0
\end{array}\right)^{k}\left(\begin{array}{l}
-1 \\
-1
\end{array}\right)+\sum_{j=0}^{k-1}\left(\begin{array}{ll}
0 & 1 \\
0 & 0
\end{array}\right)^{k-j-1}\left(\begin{array}{ccc}
1 & -1 & 2 \\
1 & 0 & -1
\end{array}\right)\left(\begin{array}{c}
(k+j)^{2} \\
k+j+3 \\
k+j)^{2}+1
\end{array}\right) \\
& =\mathcal{Z}^{-1}\left[\left(z\left(\begin{array}{ll}
1 & 0 \\
0 & 1
\end{array}\right)-\left(\begin{array}{ll}
0 & 1 \\
0 & 0
\end{array}\right)\right)^{-1}\left(z\left(\begin{array}{c}
-1 \\
-1
\end{array}\right)+\left(\begin{array}{ccc}
1 & -1 & 2 \\
1 & 0 & -1
\end{array}\right)\left(\begin{array}{c}
\frac{z^{2}+z}{(z-1)^{3}} \\
\frac{3 z^{2}-2 z}{(z-1)^{2}} \\
\frac{z^{3}-z^{2}+2 z}{(z-1)^{3}}
\end{array}\right)\right)\right] \\
& =\left(\begin{array}{c}
3 k^{2}-7 k+2-3 \delta_{0}(k) \\
-1
\end{array}\right) .
\end{aligned}
$$

Dengan menggunakan (2.14), maka solusi sistem (3.3) adalah

$$
\begin{aligned}
\mathbf{y}_{2}(k) & =-\sum_{j=0}^{h-1}\left(\begin{array}{cc}
0 & -1 \\
0 & 0
\end{array}\right)^{j}\left(\begin{array}{ccc}
0 & -1 & 0 \\
0 & 0 & 1
\end{array}\right)\left(\begin{array}{c}
(k+j)^{2} \\
k+j+3 \\
(k+j)^{2}+1
\end{array}\right) \\
& =\left(\begin{array}{c}
k^{2}+3 k+5 \\
-k^{2}-1
\end{array}\right) .
\end{aligned}
$$

Jadi, solusi sistem (3.1) adalah

$$
\mathbf{x}(k)=P \mathbf{y}(k)=\left(\begin{array}{c}
-1 \\
3 k^{2}-7 k+2-3 \delta_{0}(k) \\
-k^{2}-1 \\
-4 k^{2}+4 k-7+3 \delta_{0}(k)
\end{array}\right)
$$

\section{Ucapan Terima kasih}

Penulis mengucapkan terima kasih kepada Dr. Muhafzan, Dr. Admi Nazra, Dr. Lyra Yulianti, Dr. Dodi Devianto, dan Bapak Efendi, M.Si yang telah memberikan masukan dan saran, sehingga tulisan ini dapat diselesaikan dengan baik.

\section{Daftar Pustaka}

[1] Duan, G.R. 2010. Analysis and Design of Descriptor Linear Systems. Springer, New York

[2] Kaczorek, T. 1992. Linear Control Systems Volume 1. Research Studies Press LTD, England 
Penyelesaian Sistem Deskriptor Linier Diskrit Bebas Waktu 59

[3] Yip, E.L., and R.F. Sincovec. 1981. Solvability, controllability, and observabillity of continuous descriptor systems. IEEE Trans. Automat. Control 26 (3) : 702 706 